IP/BBSR/95-50

\title{
Spin polarisation by external magnetic fields, Aharonov-Bohm flux strings, and chiral symmetry breaking in $\mathrm{QED}_{3}$.
}

\author{
Rajesh R. Parwani \\ Institute of Physics, Bhubaneswar-751 005, \\ INDIA.ศ
}

May 1995

\begin{abstract}
In the first part, the induced vacuum spin around an Aharonov-Bohm flux string in massless three-dimensional QED is computed explicitly and the result is shown to agree with a general index theorem. A previous observation in the literature, that the presence of induced vacuum quantum numbers which are not periodic in the flux make an integralflux $\mathrm{AB}$ string visible, is reinforced. In the second part, a recent discussion of chiral symmetry breaking by external magnetic fields in parity invariant $\mathrm{QED}_{3}$ and its relation to the induced spin in parity non-invariant $\mathrm{QED}_{3}$ is further elaborated. Finally, other vacuum polarisation effects around flux tubes in different variants of QED, in three and four dimensions are mentioned.
\end{abstract}

*e-mail: parwani@iopb.ernet.in 


\section{Introduction}

Three dimensional quantum electrodynamics $\left(\mathrm{QED}_{3}\right)$, with massive electrons in an irreducible two-dimensional representation, is peculiar compared to $\mathrm{QED}_{4}$ in that it is not invariant under a discrete parity transformation. This breaking of parity by the massive fermions has a striking consequence that radiative corrections to the photon propagator generate an additional gauge-invariant but parity-odd contribution to the effective lagrangian for the gauge fields [1]. The last is the celebrated Chern-Simons term [2].

The two-component nature of the fermions, the breaking of parity by fermion masses, and the Chern-Simons term, form a triad of interlinked facts which are responsible for various interesting effects in $\mathrm{QED}_{3}$, one of which is the induction of fractional charge in the presence of a background magnetic field [1, 3]. To fix the notation, consider the lagrangian of three-dimensional QED,

$$
\mathcal{L}_{+}=\bar{\Psi}_{+}\left(i D_{\mu} \gamma^{\mu}-m\right) \Psi_{+}
$$

where $D_{\mu}=\partial_{\mu}+i e A_{\mu}, \gamma^{0}=\sigma^{3}, \gamma^{1}=i \sigma^{1}, \gamma^{2}=i \sigma^{2}$ and where $\Psi_{+}$are two component spinors. (The subscript " + " in the above equations has been inserted for latter convenience.) As mentioned, for $m \neq 0, \mathcal{L}_{+}$is not invariant 4 under the $2+1$ dimensional parity operation $(x, y) \rightarrow(-x, y), \Psi_{+} \rightarrow \sigma^{1} \Psi_{+}$. The mass parameter $m$ in (1.1) is allowed to take either sign $( \pm)$ so as to concisely describe the two inequivalent irreducible representations of $\mathrm{QED}_{3}$. If the potential $A_{\mu}$ decribes an external magnetic field with dimensionless flux

$$
F=\frac{e}{2 \pi} \oint \vec{A} \cdot d \vec{r}
$$

then the induced charge is given by

$$
\begin{aligned}
\mathcal{Q} & =\frac{e}{2} \int d^{2} x\left\langle\left[\bar{\Psi}_{+}, \gamma^{0} \Psi_{+}\right]\right\rangle \\
& =-\frac{e}{2} \int d^{2} x \sum_{E} \operatorname{sign}(E) \psi_{E}^{\dagger}(x) \psi_{E}(x) \\
& =-\operatorname{sign}(m) \frac{e F}{2} .
\end{aligned}
$$

In Eq.(1.3),$\psi_{E}$ are eigenstates of the Dirac Hamiltonian $H_{+}$, and so the induced charge is related [1, 3] to the spectral asymmetry of $H_{+}$. The last equation shows that in a time-independent external magnetic field, the asymmetry in the spectrum is an invariant depending only on the flux. There are numerous derivations of (1.4) in the literature for generic magnetic field configurations [1, 3, 5, [] and a physical picture is as follows [5]: free 
spinors have their spin correlated with their energy, $s=\frac{\operatorname{sign}(m E)}{2}$, so that a perturbing magnetic field, because of the spin-field interaction, polarises the virtual particle states differently according to $E>0$ or $E<0$ thus causing an induced charged cloud around the field.

There are other induced quantum numbers in a time-independent external magnetic field, like angular momentum [8] $\mathcal{J}$ and spin [6, 7] $\mathcal{S}$ which depend only on the flux,

$$
\begin{aligned}
\mathcal{J} & =-\operatorname{sign}(m) \frac{F^{2}}{4}, \\
\mathcal{S} & =-\operatorname{sign}(m) \frac{|F|}{4}, \text { for } m \rightarrow 0 .
\end{aligned}
$$

All of the above induced quantities depend on $\operatorname{sign}(m)$ which indicates the crucial dependence of the results on the physically different representations. Also, as required, $\mathcal{Q}$ is odd under charge conjugation (C) while $\mathcal{J}$ and $\mathcal{S}$ are C-even in agreement with the transformation properties of the corresponding single particle operators. Actually, in explicit calculations, the correct C-symmetry is ensured only if one computes properly (a)symmetrised expressions and is careful to choose regularisation schemes which preserve the (a)symmetry. Note also the very important point that while the expressions (1.4) and (1.5) for $\mathcal{Q}$ and $\mathcal{J}$ are valid in the massive theory, $\mathcal{S}$ is an invariant (depending only on the flux) only in the massless limit of the theory.

An interesting external field configuration is an Aharonov-Bohm [9] (AB) string for which the magnetic field is nonvanishing only at one point. Nevertheless, even in this singular case the relations (1.4-1.5) are still valid as has been verified by explicit calculations [10, 11, 12, 13]. In Ref. [11] it was stressed that, because of the nonperiodic (in flux) induced charge localised around an AB string in $2+1$ dimensions, an otherwise quantum mechanically unobservable string can reveal its presence to a probe electron in a Gedanken scattering experiment. This may be seen as an example in which quantum field (virtual many body) polarisation effects invalidate quantum mechanical expectations.

While the induced charge and angular momentum have been computed for an $\mathrm{AB}$ configuration, to my knowledge the induced spin has not. Other than explicitly verifying the relation (1.6) for the singular AB configuration (see Sect.2), and thereby reinforcing the last statement in the previous paragraph, the calculation involves a complication in that the limit $m \rightarrow 0$ in (1.6) must be taken before doing the space-integral over the spindensity. Actually, for an $\mathrm{AB}$ configuration, the only free dimensionfull parameter in the problem is the mass $m$ since the magnetic field enters only through the dimensionless flux $F$. Thus for the AB problem, $\mathcal{T} \equiv \int d^{2} x\left\langle\Psi_{+}^{\dagger} \frac{\sigma^{3}}{2} \Psi_{+}\right\rangle$is independent of the magnitude of $m$ 
and so trivially $\mathcal{T}^{A B}=\mathcal{T}_{m \rightarrow 0}^{A B}$. But since $\mathcal{T}$ is not related in any simple way to the spectral asymmetry, one does not expect, in general, its massless limit to coincide with $\mathcal{S}$ (that is, the massless limit required in (1.6) need not commute with doing the space-integral), and this is discussed further in Appendix A.

In $Q E D_{4}$, if the electrons are massless, the theory has a chiral symmetry. This is not true for the lagrangian (1.1) of $Q E D_{3}$ because there is no matrix which anti-commutes with the $\gamma_{\mu}$ 's. However by enlarging the representation space of the spinors in (1.1), one can also discuss chiral symmetry in massless $Q E D_{3}$. Consider thus the three space-time dimensional lagrangian given by [14]

$$
\mathcal{L}=\bar{\Psi}\left(i D_{\mu} \Gamma^{\mu}-m\right) \Psi
$$

where the four-component spinor $\Psi$ forms a reducible representation of the Dirac algebra

$$
\Gamma^{0}=\left(\begin{array}{cc}
\gamma^{0} & 0 \\
0 & -\gamma^{0}
\end{array}\right), \quad \Gamma^{1}=\left(\begin{array}{cc}
\gamma^{1} & 0 \\
0 & -\gamma^{1}
\end{array}\right), \quad \Gamma^{2}=\left(\begin{array}{cc}
\gamma^{2} & 0 \\
0 & -\gamma^{2}
\end{array}\right)
$$

with $\gamma^{0}=\sigma^{3}, \gamma^{1}=i \sigma^{1}, \gamma^{2}=i \sigma^{2}$ as before. When $m \equiv 0$, this lagrangian is invariant under a $U(2)$ symmetry generated by $I, \Gamma^{5}$, and $-i \Gamma^{3}$, where $\Gamma^{3} \equiv\left(\begin{array}{ll}0 & i \\ i & 0\end{array}\right)$ and $\Gamma^{5}=$ $i \Gamma^{0} \Gamma^{1} \Gamma^{2} \Gamma^{3}$. This symmetry is sometimes referred to as a flavour symmetry. Alternatively, since the symmetry exists only for the massless theory, one may also call it a chiral symmetry. Furthermore, by writing $\Psi \equiv\left(\begin{array}{c}\Psi_{+} \\ \Psi_{-}\end{array}\right)$, with $\Psi_{\alpha} \quad(\alpha= \pm)$ two-component spinors, the Lagrangian density (1.7) is invariant (for any $m$ ) [14 under the generalised parity operation $(x, y) \rightarrow(-x, y), \Psi_{+} \rightarrow \sigma^{1} \Psi_{-}$and $\Psi_{-} \rightarrow \sigma^{1} \Psi_{+}$.

As (1.7) has symmetries similar to those of physically interesting, but technically difficult, four-dimensional theories like QCD, it has been used as a toy model to study such issues as chiral symmetry breaking. Most of the last work has been on dynamical chiral symmetry breaking, which involves the difficult problem of finding self-consistent nonperturbative solutions to the Schwinger-Dyson equations 14. A simpler, but still informative, task is to study chiral symmetry breaking induced by external electromagnetic fields. In Ref.[15] it was found that a space-time independent external magnetic field yielded a nonzero value for the chiral order parameter, $\left\langle\bar{\Psi}_{+} \Psi_{+}\right\rangle_{m \rightarrow 0}$. This result was generalised to inhomogeneous (but still static) fields in Ref. [16] by observing that in an external field (that is ignoring virtual photon corrections), the chiral condensate of the lagrangian $\mathcal{L}$ is related to the induced spin density of the lagrangian $\mathcal{L}_{+}$. In terms of two-component spinors, Eq.(1.7) may be written as

$$
\mathcal{L}=\sum_{\alpha= \pm} \mathcal{L}_{\alpha} \equiv \sum_{\alpha= \pm} \bar{\Psi}_{\alpha}\left(i D_{\mu} \gamma^{\mu}-\alpha m\right) \Psi_{\alpha}
$$


so that if $A_{\mu}$ is an external field, (11.9) describes two decoupled systems $\mathcal{L}_{ \pm}$. The fermion condensate is therefore

$$
\begin{aligned}
C(x ; m) \equiv\langle\bar{\Psi}(x) \Psi(x)\rangle & =\sum_{\alpha= \pm} \alpha\left\langle\bar{\Psi}_{\alpha}(x) \Psi_{\alpha}(x)\right\rangle_{\alpha} \\
& \equiv \sum_{\alpha= \pm} \alpha C_{\alpha}(x ; m) \\
& =\sum_{\alpha= \pm} \alpha C_{+}(x ; \alpha m),
\end{aligned}
$$

where $\left\langle\bar{\Psi}_{+} \Psi_{+}\right\rangle_{+}$denotes the expectation value of $\bar{\Psi}_{+} \Psi_{+}$in the $\mathcal{L}_{+}$subsystem. Thus in an external field the properties of the condensate $C(x ; m \rightarrow 0)$ are determined completely by the induced spin density $\frac{1}{2} C_{+}=\left\langle\Psi_{+}^{\dagger} \frac{\sigma^{3}}{2} \Psi_{+}\right\rangle_{+}$in the subsystem $\mathcal{L}_{+}$which is a better studied problem. An immediate consequence of $(1.12)$ is $C(x ;-m)=-C(x ; m)$, so that the condensate $C(x ; m \rightarrow 0)$ can be nonzero only if the massless limit is discontinuous, that is it depends on $m \rightarrow 0^{ \pm}$. That this is indeed the case was noted in Ref. 16 and Sect.(3) of this paper extends the discussion of chiral-symmetry breaking given there.

The plan of the paper is as follows. In Section 2.1 a derivation of the relation (1.6) is reviewed and in Sect. 2.2 it is verified by an explicit calculation in the AB system. Section 3 repeats some of the discussion on chiral symmetry breaking given in Ref.[16] and supplements it with some qualitative comments. The concluding section of this paper summarises some of the discussion and highlights other vacuum polarisation effects in $Q E D_{3}$ and $Q E D_{4}$. The appendices contain some details of the calculations presented in Sect.2. Appendix B also presents a novel derivation of (1.4) for the AB case in the massless limit. The reader is informed that for continuity of discussion, some material from the short report [16] has been reproduced in this article.

\section{Induced Spin}

\section{$2.1 \quad$ General}

Consider the induced vacuum spin in the theory defined by (1.1), due to vacuum polarisation in a time-independent external magnetic field,

$$
\frac{1}{2} C_{+}(x ; m \rightarrow 0)=\lim _{m \rightarrow 0}\left\langle\Psi_{+}^{\dagger} \frac{\sigma^{3}}{2} \Psi_{+}\right\rangle .
$$

Since $m$ can be of either sign, the limit in (2.1) should be understood correspondingly as $m \rightarrow 0^{ \pm}$. On expanding the fermion operators in terms of the eigenstates $\psi_{E}$ of the Dirac Hamiltonian $H_{+}=\gamma^{0} \gamma^{i}\left(p^{i}-e A^{i}\right)+m \gamma^{0}$ (in the $A_{0}=0$ gauge), symmetrising 
with respect to charge-conjugation, and subtracting the infinite vacuum contribution, one obtains

$$
\frac{1}{2} C_{+}(x ; m \rightarrow 0)=-\left.\frac{1}{4} \lim _{m \rightarrow 0} \sum_{E} \operatorname{sign}(E) \psi_{E}^{\dagger} \sigma^{3} \psi_{E}\right|_{0} ^{F}
$$

The summation in (2.2) symbolically denotes a sum over discrete states and an integral over the continuum. In the $m \rightarrow 0$ limit, the $E>0$ and $E<0$ eigenstates are related 17] by $\psi_{-E}=\sigma^{3} \psi_{E}$, so that only the zero modes contribute to (2.2). For $F>0$ the zero modes occur at $E=m \rightarrow 0$ and are of the form $\psi_{0} \sim\left(\begin{array}{l}u \\ 0\end{array}\right)$, while for $F<0$ they occur at $E=-m \rightarrow 0$ and are of the form $\psi_{0} \sim\left(\begin{array}{l}0 \\ v\end{array}\right)$. Denote by $I$ the smallest integer greater than or equal to $|F|-1$; then for a given $m=0^{ \pm}$, there are $I$ discrete (normalisable to unity) states in addition to the continuum (scattering) states 17, 5. Eq. 2.2) therefore becomes

$$
\frac{1}{2} C_{+}\left(x ; m \rightarrow 0^{ \pm}\right)=-\left.\frac{\operatorname{sign}(m)}{4} \sum_{E=m \rightarrow 0^{ \pm}} \psi_{E}^{\dagger} \psi_{E}\right|_{0} ^{F}
$$

The discrete zero modes in the sum (2.3) are localised [17, 5] around the magnetic field and so give a nonzero local contribution. On the other hand the continuum zero modes, being scattering states, give a negligible local contribution [7] unless the flux is infinite. However the continuum states are important to establish the index theorem which is obtained by integrating (2.3) over all space : The discrete states contribute an amount $-\frac{\operatorname{sign}(m)}{4} I$, while a careful analysis [7] shows that the scattering states contribute $-\frac{\operatorname{sign}(m)}{4}(|F|-I)$,

$$
\mathcal{S} \equiv \int d^{2} x \frac{C_{+}\left(x ; m \rightarrow 0^{ \pm}\right)}{2}=-\frac{\operatorname{sign}(m)}{4}|F| .
$$

The discussion above has been for regular field configurations. If the flux contracts to a single point to form an AB flux string then no discrete normalisable states form even for $|F|>1$ because of the highly singular nature of the field. Rather, if one starts from a finite size tube and shrinks it to zero, the would be discrete states collapse to point-like states located on the string. These hidden states maintain the relations (1.4, 1.5, 1.6) in the $\mathrm{AB}$ case when $|F|>1$ but then they cause a breakdown in periodicity, with respect to the flux, of induced quantum numbers in that configuration 11, 12.

\subsection{The AB case}

The purpose here is to verify (2.4) explicitly for an $\mathrm{AB}$ string with $|F|<1$. The eigenstates of the $\mathrm{AB}$ hamiltonian in polar coordinates, and in the $A_{0}=0, e \vec{A}=\frac{F}{r} \hat{\theta}$ gauge, are 
readily determined,

$$
\begin{gathered}
\psi_{E, n}=\left[\frac{(E-m) k}{4 \pi E}\right]^{\frac{1}{2}}\left(\begin{array}{c}
\left.\frac{k e^{i(n-1) \theta}}{E-m} \epsilon_{n} J_{\epsilon_{n}(l-1)}(k r)\right), \\
e^{i n \theta} J_{\epsilon_{n} l}(k r)
\end{array}\right) \\
l \equiv n-F, \quad n=\text { integer, }|F|<1, F \neq 0, \\
E= \pm \sqrt{k^{2}+m^{2}}, \quad 0 \leq k \leq \infty, m \neq 0, \\
\epsilon_{n}=+1 \text { for } n \geq 1 \quad \text { and } \quad \epsilon_{n}=-1 \text { for } n \leq 0 .
\end{gathered}
$$

In the above, the sign of $\epsilon_{n}$ has been determined by the usual requirement of squarenormalisability [18 of the wavefunctions and a possible ambiguity for the critical partial wave $0<l_{c}<1$ is resolved by considering the string to be a limiting case of a flux tube of finite size [9]. Notice that the critical partial wave spinor contains one component which is singular at the origin. Also note that the values $F=0, \pm 1$, which have been excluded from (2.5) for technical convenience, can be obtained in matrix elements (the induced charge, spin etc.) by taking the limits $F \rightarrow 0, \pm 1$.

Substituting (2.5) into the right-hand-side of (2.2) and integrating over all space gives

$$
\mathcal{S}=\frac{-1}{8 \pi} \int d^{2} x\left(\left.m \sum_{n} \int_{0}^{\infty} \frac{d k k}{\sqrt{k^{2}+m^{2}}}\left\{J_{\epsilon_{n}(l-1)}^{2}+J_{\epsilon_{n}(l)}^{2}\right\}\right|_{0} ^{F}\right)_{m \rightarrow 0}
$$

where the massless limit has to be taken inside the space-integral. Before evaluating this quantity, consider first the same expression but with the massless limit taken after doing the spatial integral. Calling this last object $\mathcal{T}_{m \rightarrow 0}^{A B}$, one has

$$
\mathcal{T}^{A B}=-\left.\frac{m}{8 \pi} \int d^{2} x \int_{0}^{\infty} \frac{d k k}{\sqrt{k^{2}+m^{2}}} \sum_{n \geq 0} G_{n}(k r)\right|_{0} ^{F}
$$

where

$$
G_{n}(k r) \equiv J_{n-F}^{2}(k r)+J_{n+F}^{2}(k r)+J_{n-F+1}^{2}(k r)+J_{n+F+1}^{2}(k r)
$$

The quantity $\mathcal{T}^{A B}$ is discussed at length in Appendix A.

Let us now return to the evaluation of (2.6), that is, of $\mathcal{S}$. For this we need the massless limit of the wavefunctions in (2.5). It is easy to verify that for $m \equiv 0, \psi_{-k, n}=-\psi_{k, n}$ and if this is used indiscrimately in (2.2) one obtains zero for the induced spin. The 
resolution of this paradox lies in recalling that there ought to be unpaired continuum zero modes $\left(E=0^{ \pm}\right)$. In order to isolate these unambiguously one has to start with $m \neq 0$. Now when $m \neq 0$ (that is before the massless limit), the states with energy $E \sim \pm m$ correspond to values of $k$ satisfying $k \ll|m|$. Thus one considers the $\frac{k}{|m|} \rightarrow 0$ limit in (2.5) to identify the nonvanishing threshold states. In this limit only the critical partial wave survives: For $1>F>0$ its limiting form is

$$
\lim _{k \rightarrow 0} \Psi_{n_{c}=1}=\operatorname{sign}(m) \sqrt{\frac{k}{2 \pi}}\left(\begin{array}{c}
J_{-F}(k r) \\
0
\end{array}\right), E \approx m, F>0,
$$

while for $0>F>-1$ it is

$$
\lim _{k \rightarrow 0} \Psi_{n_{c}=0}=\sqrt{\frac{k}{2 \pi}}\left(\begin{array}{c}
0 \\
J_{F}(k r)
\end{array}\right), E \approx-m, F<0 .
$$

(The Bessel function $J_{|F|}(k r)$ has not been expanded in $k$ because $k r$ can be large even if $k$ is small). When finally the limit $m \rightarrow 0$ is taken, these (2.9-2.10) will be the only states which will contribute to the spin, the contribution from the $|E|>0$ states cancelling in pairs as mentioned above. Thus for $|F|<1$ the [19 scattering zero modes 2.9 2.10) contribute to (2.2) the amount

$$
\begin{aligned}
\frac{1}{2}\left\langle\bar{\Psi}_{+} \Psi_{+}\right\rangle_{m \sim 0} & =-\left.\frac{\operatorname{sign}(m)}{4} \int_{0}^{\lambda} \frac{d k}{2 \pi} k J_{-|F|}^{2}(k r)\right|_{0} ^{F} \\
& =-\left.\frac{\lambda^{2} \operatorname{sign}(m)}{16 \pi}\left\{J_{-|F|}^{2}(\lambda r)-J_{-|F|-1}(\lambda r) J_{-|F|+1}(\lambda r)\right\}\right|_{0} ^{F},
\end{aligned}
$$

where $\lambda \ll|m|$. Note that taking the limit $|m| \rightarrow 0$ (and so $\lambda \rightarrow 0$ ) on the right-handside of (2.12) results in a null contribution to the (local) spin-density in accordance with the remarks in the previous subsection that for $|F|<1$ only the dilute continuum zero modes contribute to the density. However the space-integral of the density in (2.12) is non-negligible and is given by

$$
\begin{aligned}
2 \pi \operatorname{sign}(m) \int_{0}^{\infty} \frac{d r}{2} r\left\langle\bar{\Psi}_{+} \Psi_{+}\right\rangle_{m \sim 0} & =-\left.\frac{\lambda^{2} \delta(0)}{8 \lambda}\right|_{0} ^{F}+\left.\frac{\lambda^{2}}{8} \int_{0}^{\infty} d r r\left\{J_{-|F|-1}(k r) J_{-|F|+1}(k r)\right\}\right|_{0} ^{F} \\
& =\left.\frac{1}{8} \int_{0}^{\infty} d x x\left\{J_{-|F|-1}(x) J_{-|F|+1}(x)\right\}\right|_{0} ^{F} \\
& =\frac{1}{8}(-2|F|) \\
& =-\frac{|F|}{4}
\end{aligned}
$$


and so

$$
2 \pi \int_{0}^{\infty} d r \frac{r}{2}\left\langle\bar{\Psi}_{+} \Psi_{+}\right\rangle_{m \rightarrow 0}=-\operatorname{sign}(m) \frac{|F|}{4}, \quad|F|<1,
$$

which agress with the index theorem (1.6). Note that the integral in (2.13) is independent of the parameter $\lambda$ so that the final result is not sensitive to the slight ambiguity in the choice of this cutoff. The evaluation of the integral (2.13) is described in Appendix B.

To summarise the results of this section, the induced spin in the AB system was computed for $|F|<1$ and the index formula (1.6) verified. It was shown that isolating the contribution of the threshold states required a careful consideration of the massless limit. For $|F|<1$ the contribution to $\mathcal{S}$ is smeared over all space due to the nonlocalised nature of the continuum (scattering) threshold states and thus the spin-density (in the required massless limit) is zero. For $|F|>1$ there are also discrete states for any finite size flux tube which give a localised nonzero contribution to $\mathcal{S}$ (see the analogous discussion for the charge in [11, 12]). As mentioned earlier, these discrete states collapse to point-like states in the limit of a zero-radius flux tube.

The presence of induced quantum numbers around the $Q E D_{3} \mathrm{AB}$ string implies that it is detectable (through a scattering experiment) even for integral flux [11]. However, there is a return flux at infinity (actually, at $r \sim 1 / \mu$ where $\mu \sim e^{2}$ is the dynamically generated photon mass) complementary to the $\mathrm{AB}$ flux in the string, and one can ask if the vacuum polarisation effects associated with that return flux can aid in restoring the invisibility of the integral flux string. If the mass $m$ of the electron is much larger than $\mu$, then for any $F$ the polarisation effects (1.4-1.5) are localised within $1 / m$ of the string and are probably not significantly [11] affected by the return flux effects located at $1 / \mu$ . For $F$ exactly an integer the induced effects are exactly on the string and so should be even more immune from the return flux effects.

The situation is more complicated for massless electrons, which is the limit in which the induced spin (1.6) is defined. However even in the massless case, the contribution from the collapsed threshold states (for $|F|>1$ ) is highly localised so it is not very clear to me if these bound states will be completely nullified in their contribution to (1.4 1.6) by feedback effects. One does however expect the contribution from the scattering states to be cancelled by feedback [20].

There are other subtleties associated with the massless theory. For example, the factor of $\operatorname{sign}(m)$ in (2.14), if averaged over, suggests no polarisation effects in the massless limit. However if one adopts the point of view that the theory is sensibly defined only for massive electrons (to define an unambiguos zero of energy) then no matter how small that mass is 
with respect to $e^{2}$, the $\operatorname{sign}(m)$ factor distinguishes between the two inequivalent physical representations one is working in and so should not be averaged over. Indeed, even if one starts with $m \equiv 0$ in (1.7), a small nonperturbative mass is generated dynamically [14. This suggests that the ambiguities of a truly massless theory are probably academic and one should at most speak of a massless limit, where the fermion mass is very much smaller than the other dimensionfull parameter, $e^{2}$.

The discussion in this section has been for the parity non-invariant lagrangian (1.1). In the parity-invariant theory (1.7 1.9) there is no photon mass generation due to cancellations between the contributions of the "+" and "-" spinors, and so no induced charge like (1.4).

\section{Chiral symmetry breaking}

Let $q$ be the Dirac fermion operator in four space-time dimensions. Then to study dynamical chiral symmetry breaking, one looks at the condensate $<\bar{q} q>$ in the massless limit of the full dynamical theory. In general this is difficult to do analytically and an intermediate step that can give some insight is to look at the condensate for a particular background gauge-field. In Ref. 21] it was shown that if $\left\langle\bar{q} q>^{A}\right.$ is the space-time average of the condensate in the background field $A_{\mu}$, and $\rho_{D}(\lambda)$ is the density of eigenvalues of the background operator $i \not D$ per unit space-time volume, then

$$
<\bar{q} q>^{A}=\pi \rho_{D}(0) .
$$

That is, chiral symmetry breaking in a given background field is related to the spectral density at zero energy of the operator $i \not D$. If one can parametrize the right-hand-side of (3.1) explicitly in terms of the background field $A_{\mu}$, then by integrating (in the pathintegral) Eq.(3.1) over all possible gauge-field configurations, one can in principle study dynamical chiral symmetry breaking in the full theory. In practice however, it is already quite difficult to achieve the first step above : that is, to make statements about (3.1) for arbitrary configurations.

However for three-dimensional QED described by the parity-invariant lagrangian (1.7), much can be said. For external time-independent magnetic fields with flux $F$, the condensate is given by $(1.12-2.3)$ to be

$$
C\left(x ; m \rightarrow 0^{ \pm}\right)=-\left.\operatorname{sign}(m) \sum_{E=m \rightarrow 0^{ \pm}} \psi_{E}^{\dagger} \psi_{E}\right|_{0} ^{F},
$$

where $\psi_{E}$ are the eigenstates discussed in the previous section. It is quite clear that (3.2) is just a special case of (3.1), obtained by restricting to time-independent magnetic fields. 
However (3.2) contains some unique features which enhance its usefulness in spite of the restrictions. For any finite flux, the spectral density on the right-hand-side of (3.2) is dominated locally by a finite number of discrete (normalisable to unity) zero modes. The number of such modes is determined solely by the flux as discussed earlier. Furthermore, the integrated density also depends only on the flux,

$$
\int_{0}^{\infty} d^{2} x\left\langle\bar{\Psi}_{+} \Psi_{+}\right\rangle_{m \rightarrow 0}=-\operatorname{sign}(m)|F| .
$$

This last is significant for it means that for any configuration with nonzero net flux, the chiral condensate must be nonzero at some place. Thus one can decide if chiral-symmetry is broken or not by just knowing the flux rather than the details of the magnetic field. This also suggests that the contribution of static magnetic fields to the full dynamical condensate is not negligible (of nonzero measure in the path-integral) and makes it plausible that dynamical chiral symmetry happens in the full dynamical theory (that is, without external fields). Of course the last is already known to happen [14] and so the real challenge is to see if one can use (3.2 3.3$)$, integrated over arbitary static magnetic fields (of variable fluxes) to get an estimate for the dynamical condensate. By ignoring time-dependent fields, and in particular electric fields, there is no a priori reason such an estimate would be close to the results of Ref. [14, but still the exercise might be revealing if doable. (This will not be attempted here.)

Another important feature of (3.2) and (3.3) is the $\operatorname{sign}(m)$ factor. As noted in Ref. [16] this means that the direction of chiral symmetry breaking in external magnetic fields is correlated with the infinitesimal breaking of that symmetry brought about by a mass $m=0^{ \pm}$(see the discussion of massless theories near the end of the last section).

Let me mention now some perturbations which could destroy the condensate (3.2 3.3) formed by static magnetic fields. Viewing the condensate as an ordering of spins in a magnetic field, one expects a superimposed electric field to reduce such an ordering and so cause a reduction in the magnitude of the condensate. At least if the electric field is weak then one might be able to check this in a perturbative treatment as was done in Ref. 22] for a Chern-Simons theory.

Thermal fluctuations are also naturally expected to disrupt any ordering (symmetry breaking). For example, the charge (1.4) has been computed [3, 23] for slowly varying magnetic fields at nonzero temperature and density, showing a rapid "washing out" at high temperature. More remarkably, for any non-zero temperature, the charge was shown to vanish in the massless limit; a simple explanation of this given in [3] was that in the massless theory the charge is due to the zero-energy modes which are susceptible to large 
fluctuations in their occupancy at nonzero temperature. Since the chiral condensates (3.2-3.3) also receive support only from the zero modes, the qualitative argument given above suggests that they should also vanish at nonzero temperature. This has recently been noted in an explicit calculation for a constant magnetic field [24].

\section{Conclusion}

In this paper were studied some phenomena in three-dimensional QED in an external magnetic field. The breaking of chiral symmetry by an external magnetic field in the reducible representatiuon of $\mathrm{QED}_{3}(1.7)$ is equivalent to the polarisation of spin in the irreducible theory (1.1). Thus chiral symmetry breaking in the toy-model (1.7) may be heuristically understood as an ordering of spins in a magnetic field. Then any effect which interferes with this ordering, such as an electric field or thermal fluctuations, would be expected to diminish the breaking of chiral symmetry.

Quantitatively, the chiral condensate in an external magnetic field is controlled by the total flux of that field (3.2) as it determines the number and nature (discrete and localised versus continuum and non-localised) of the zero modes of the massless Dirac equation. For any finite flux, the localised zero modes give the essential non-negligible contribution to the chiral-condensate. The space-integral of the condensate receives a nontrivial contribution also from the dilute scattering zero modes and the net result is an invariant depending only on the flux (3.3). This index formula gives the phenomena a certian robustness and might be useful in obtaining estimates of the chiral condensate in the full dynamical theory as described in Sect.3.

The polarisation effects (1.4, 1.5, 1.6) in the theory (1.1) are also invariants depending only on the flux. It has been verified ([10, 11, 12, 13] and Sect.2) that the formulae (1.4, 1.5, 1.6) hold also for singular configurations such as the AB flux string. Consequently, as a result of the localised threshold states which form every time the flux passses an integer, there accumulate around an $\mathrm{AB}$ string vacuum polarisation effects which are not periodic in the flux. This exposes an integral flux $(F \neq 0)$ string to external probes [11]. By contrast, such polarisation effects cancel for flux tubes in $\mathrm{QED}_{4}$ due to the four component nature of the fermions which preserve parity [26, 12]. However this does not rule out other polarisation effects (as yet undiscovered) which might also make an integral $\mathrm{AB}$ flux string in $\mathrm{QED}_{4}$ visible.

In the model (1.7) the net induced charge around a magnetic field vanishes because the contribution from the "+ and " - "two-component spinors cancel (since they 
have opposite signs of mass). One can consider a modification of (1.7) which is also parity invariant but in which the net induced charge is nonzero. This model is obtained by replacing the charge $e$ in $(1.7)$ by the matrix $e^{\star}=e\left(\begin{array}{cc}I & 0 \\ 0 & -I\end{array}\right)$. This means that the \pm spinors now have opposite charges in addition to oppositely signed masses and their respective contribution to the induced charge will be additive. This last model has actually been used as a parity-invariant effective lagrangian for high-temperature superconductivity, but the gauge-field which couples to the charge $e^{\star}$ there is a "statistical gauge field" 25] rather than the true electromagnetic field.

There certainly exist vacuum polarisation effects around flux tubes, even in parity-odd three-dimensional QED (1.1), which are not caused by parity breaking effects like fermion masses. For example, there are vacuum currents which circulate around thin magnetic flux tubes and are due to the $A B$ effect acting on virtual electron-positron pairs [26, 11, 12]. These currents occur also for scalar theories and in $3+1$ dimensions. A possible role for them has been proposed recently in a picture of a nonperturbative vacuum of $\mathrm{QED}_{4}$ 27, 28.

Whether one uses three-dimensional QED as a simplified model in particle physics, as an effective theory in condensed matter physics, or as a field theory of intrinsic interest, it is clear that much more remains to be understood. The focus in this paper has been on extending previous discussions of polarisation effects due to magnetic fields. The interested reader is referred to some other recent works [29] in which magnetic fields are also employed to unravel the dynamics of $\mathrm{QED}_{3}$.

\section{Acknowledgements}

I thank Alfred Goldhaber and Hsiang-nan Li for helpful comments, and Pijush Ghosh for Ref. [22]. 


\section{Appendix A}

Here the quantity $\mathcal{T}^{A B}$ mentioned in Sect.2 is evaluated, and some subtleties involved are discussed by comparing the calculation with the corresponding one for the induced charge $\mathcal{Q}$.

Simplifying $\left\langle\bar{\Psi}_{+} \Psi_{+}\right\rangle_{+}$for the AB case one obtains,

$$
\begin{aligned}
\left\langle\bar{\Psi}_{+} \Psi_{+}\right\rangle_{+} & =\frac{-1}{4 \pi}\left(\left.m \sum_{n} \int_{0}^{\infty} \frac{d k k}{\sqrt{k^{2}+m^{2}}}\left\{J_{\epsilon_{n}(l-1)}^{2}+J_{\epsilon_{n}(l)}^{2}\right\}\right|_{0} ^{F}\right) \\
& =-\left.\frac{m}{4 \pi} \int_{0}^{\infty} \frac{d k k}{\sqrt{k^{2}+m^{2}}} \sum_{n \geq 0} G_{n}\right|_{0} ^{F}
\end{aligned}
$$

where

$$
G_{n}(k r) \equiv J_{n-F}^{2}(k r)+J_{n+F}^{2}(k r)+J_{n-F+1}^{2}(k r)+J_{n+F+1}^{2}(k r)
$$

Note the manifest C-symmetry, $F \rightarrow-F$, of the expression for $G_{n}$. By writing

$$
\frac{1}{\sqrt{k^{2}+m^{2}}}=\frac{2}{\sqrt{\pi}} \int_{0}^{\infty} d \alpha e^{-\alpha^{2}\left(k^{2}+m^{2}\right)}
$$

the $k$-integral in (A.2) may be performed using [30]

$$
\int_{0}^{\infty} e^{-\alpha^{2} k^{2}} J_{\mu}^{2}(k r) k d k=\frac{1}{2 \alpha^{2}} e^{-\frac{r^{2}}{2 \alpha^{2}}} I_{\mu}\left(\frac{r^{2}}{2 \alpha^{2}}\right),
$$

to give

$$
\left\langle\bar{\Psi}_{+} \Psi_{+}\right\rangle_{+}=-\left.\frac{m}{4 \pi \sqrt{\pi}} \int_{0}^{\infty} \frac{d \alpha}{\alpha^{2}} e^{-\alpha^{2} m^{2}-z}\left(\sum_{n \geq 0} H_{n}(z)\right)\right|_{0} ^{F}
$$

where

$$
H_{n}(z) \equiv I_{n-F}(z)+I_{n+F}(z)+I_{n-F+1}(z)+I_{n+F+1}(z)
$$

and $z=r^{2} / 2 \alpha^{2}$. Now consider the spatial $\int r d r d \theta$ integral over the density in (A.6). The $\alpha$-integral can then be done explicitly because it reduces to the simple Gaussian

$$
\int_{0}^{\infty} d \alpha e^{-\alpha^{2} m^{2}}=\frac{\sqrt{\pi}}{2|m|}
$$

after the change of variable $r^{2}=2 \alpha^{2} t$ in the $r$-integral. Thus one is left with

$$
\int d^{2} x\left\langle\bar{\Psi}_{+} \Psi_{+}\right\rangle_{+}=-\left.\frac{\operatorname{sign}(m)}{4} \int_{0}^{\infty} d t e^{-t}\left(\sum_{n \geq 0} H_{n}(t)\right)\right|_{0} ^{F} .
$$


Assuming that the order of integration in (A.9) may be interchanged with the summation, the $t$-integral may be evaluated by taking appropriate limits of the formula [30]

$$
\int_{0}^{\infty} e^{-a t} I_{p+\nu}(b t)=\frac{b^{p+\nu}}{\sqrt{a^{2}-b^{2}}\left(a+\sqrt{a^{2}-b^{2}}\right)^{p+\nu}} .
$$

For example, for any integer $n$,

$$
\int_{0}^{\infty} e^{-t}\left(I_{n+F}(t)-I_{n}(t)\right)=-F .
$$

Thus the final result for the space-integral of (A.1) is

$$
\int d^{2} x\left\langle\bar{\Psi}_{+} \Psi_{+}\right\rangle_{+}=-\frac{\operatorname{sign}(m)}{4} \sum_{n \geq 0}(F-F+F-F)=0 .
$$

Therefore $\mathcal{T}^{A B}=\frac{1}{2}$ (Eq. A.12) $)=0$, if the space-integral above may be performed before the infinite partial wave summation.

By way of comparison consider the charge (1.3) evaluated for the AB case with $|F|<1$. Substituing (2.5) into ([1.3) and simplifying gives [11],

$$
\begin{aligned}
\mathcal{Q} & =\frac{-m e}{4 \pi} \int d^{2} x\left(\sum_{n} \int_{0}^{\infty} \frac{d k k}{\sqrt{k^{2}+m^{2}}}\left\{J_{\epsilon_{n}(l-1)}^{2}-J_{\epsilon_{n}(l)}^{2}\right\}\right) \\
& =\frac{-m e \operatorname{sign}(F)}{4 \pi} \int d^{2} x\left(\int_{0}^{\infty} \frac{d k k}{\sqrt{k^{2}+m^{2}}}\left\{J_{-|F|}^{2}-J_{|F|}^{2}\right\}\right) \\
& =-\operatorname{sign}(m) \frac{e F}{2} .
\end{aligned}
$$

Apart from a factor $2 e$, the expression (A.13) differs from $\mathcal{T}^{A B}$ by a relative minus sign between the two Bessel functions in curly brackets. Consequently the sum over partial waves in (A.13) results in several cancellations to give (A.14). By writing $J_{|F|}-J_{-|F|}=$ $\left(J_{|F|}-J_{0}\right)-\left(J_{-|F|}-J_{0}\right)$, Eq. (A.14) may be evaluated using (A.4, A.5, A.11) to get (A.15). (This computation is slightly different from the one in Ref. 111 where (A.11) was not used).

Since $\mathcal{Q}$ is C-odd no $F=0$ subtraction is necessary in (A.13) as that piece is automatically zero. On the other hand since $\mathcal{T}($ and $\mathcal{S}$ ) are C-even, a $F=0$ subtraction is necessary to remove an infinite bare vacuum contribution. The last infinity is due to the fact that the spin of free fermions is given by $s=\frac{1}{2} \operatorname{sign}(m E)$ so that the spin of the bare ground state, $\sum_{E} \operatorname{sign}(E) s \sim \sum \operatorname{sign}(m)$, is divergent. 
If the inifinite summation over partial waves in (A.13) is regulated by a cut-off, $|n|_{\max }=N,(N>0)$ which is later taken to infinity, one obtains (see [20]),

$$
\begin{aligned}
\mathcal{Q} & =\frac{-m e}{4 \pi} \lim _{N \rightarrow \infty} \int d^{2} x\left(\sum_{-N}^{N} \int_{0}^{\infty} \frac{d k k}{\sqrt{k^{2}+m^{2}}}\left\{J_{\epsilon_{n}(l-1)}^{2}-J_{\epsilon_{n}(l)}^{2}\right\}\right) \\
& =\frac{-m e}{4 \pi} \lim _{N \rightarrow \infty} \int d^{2} x \int_{0}^{\infty} \frac{d k k}{\sqrt{k^{2}+m^{2}}}\left(\left\{J_{-F}^{2}-J_{F}^{2}\right\}+\left\{J_{N-F}^{2}-J_{N+1+F}^{2}\right\}\right)
\end{aligned}
$$

It is easy to verify using the formulae given earlier that if the limit $N \rightarrow \infty$ in (A.17) is taken after the space-integral, the answer is zero as the contribution from the $N$-dependent Bessel functions cancels the contribution from the first two. However, as noted in Ref. 20], in the large $N$ limit the contribution from the $N$-dependent pieces recedes to infinity and it may be interpreted as representing the oppositely charged polarisation cloud "repelled" by the localised flux. So if one is interested in the local polarisation effects, the $N \rightarrow \infty$ limit should be taken before the space-integral in (A.17). Note also that for any finite $N$ the expression (A.17) is no longer C-odd, this asymmetry being broken by the $N$ dependent pieces. This may be remedied by taking the C-odd part of the truncated sum in (A.16). The rest of the discussion in this paragraph still goes through then.

Returning now to the evaluation of $\mathcal{T}^{A B}$, consider regulating the partial-wave sum (A.9) in a $C$-even way as for the charge above. Clearly, the result is still zero if the space-integral is done first before the summation. This suggests, by analogy with the charge case above, that in this order (space-integral before summation) of calculating $\mathcal{T}$ one is adding the local polarisation effects to the opposite non-local ones. Unfortunately, because there are no pair-wise cancellations among the partial waves in (A.9) as in the charge case, it is not obvious which of the partial waves are contributing to the local polarisation effects and which pieces may be interpreted as the opposite effects which recede to infinity in the $N \rightarrow \infty$ limit. Fortunately however this question, though interesting, is not of direct relevance because the physical object $\mathcal{S}$ as defined in (2.4) differs from $\mathcal{T}$ in that the massless limit has to be taken before the space-integral (as suggested by the general derivation sketched in Sect.2 ). The evaluation of $\mathcal{S}$ is described in the main text. 


\section{Appendix B}

Here the integral in Eq. 2.13) is evaluated. It is

$$
\begin{aligned}
& \int_{0}^{\infty} d x x\left(J_{-|F|-1}(x) J_{-|F|+1}(x)-J_{-1}(x) J_{1}(x)\right) \\
&=\int_{0}^{\infty} d x x\left(J_{-|F|-1}(x) J_{-|F|+1}(x)+J_{1}^{2}(x)\right)
\end{aligned}
$$

with $|F|<1$. The integral (B.1) is well defined but inconvenient to evaluate as a whole. Therefore the integral over the $F$-dependent and $F$-independent Bessel functions in (B.1) will be performed separately. This will require an intermediate regularisation. The method adopted here is the following. One has an integral [30]

$$
\begin{aligned}
\int_{0}^{\infty} J_{\mu}(t) J_{\nu}(t) t^{-\alpha} d t= & \frac{\Gamma(\alpha) \Gamma\left(\frac{\nu+\mu-\alpha+1}{2}\right)}{2^{\alpha} \Gamma\left(\frac{-\nu+\mu+\alpha+1}{2}\right) \Gamma\left(\frac{\nu+\mu+\alpha+1}{2}\right) \Gamma\left(\frac{\nu-\mu+\alpha+1}{2}\right)} \\
\text { with } & \nu+\mu+1>\alpha>0
\end{aligned}
$$

By setting $\mu=-|F|-1$ and $\nu=-|F|+1$ in $(\underline{B .2})$ and choosing $F$ and $\alpha$ so that the condition $(\overline{\mathrm{B} .3})$ is satisfied, the $F$ dependent integral in ( $\mathrm{B} .1)$ may be performed by analytically continuing the right-hand-side of $(\mathbb{B . 2})$ to $\alpha=-1$ and the full range of $F$. The $F$-independent integral in (B.1) may be evaluated similarly. The net result for (B.1) is $-2|F|$.

It is useful to have a cross-check on the above manipulations with (B.2). For this purpose, consider the following calculation of the charge (1.3), for the AB system with flux $|F|<1$, in the massless limit of the theory. Using the continuum threshold states given by $2.9-2.10)$ and the mapping $\psi_{E}=\sigma^{3} \psi_{E}$ between the non-threshold states, one obtains (cf. the calculation of the spin in 2.12-2.14)

$$
\begin{aligned}
\lim _{m \rightarrow 0} \mathcal{Q} & =\left.\frac{-e}{2} \operatorname{sign}(m) \operatorname{sign}(F) \lim _{\lambda \rightarrow 0} \int_{0}^{\infty} 2 \pi r d r \int_{0}^{\lambda} \frac{d k}{2 \pi} J_{-|F|}(k r)\right|_{0} ^{F} \\
& =\frac{-e}{2} \operatorname{sign}(m) \operatorname{sign}(F)|F| \\
& =\frac{-e}{2} \operatorname{sign}(m) F
\end{aligned}
$$

where $\lambda \ll|m|$. In (B.4) a $F=0$ subtraction has been inserted to remove divergent $F$-independent pieces. Such a subtraction was not required in the calculation of $Q$ (for $m \neq 0$ ) by the conventional method in Appendix A (see A.13) and so presumably it is 
required here because of the ambiguity in the cut-off $\lambda$. The integral in (B.4) has been evaluated using (B.2) and the final result (B.6) agrees with the expected formula (1.4) which holds independent of the magnitude of $m$. (Please note however that the chargedensity is not a topological invariant and it also depends nontrivially on the mass $m$. Thus, for example, for $|F|<1$ the charge-density is infinitely dilute in the $m \rightarrow 0$ limit (receiving contributions only from continuum zero modes (B.4)) but is concentrated within a distance $1 / m$ of the localised field for $m \neq 0$ (in which case it receives contributions also from non-threshold continuum states) [11, 12].) 


\section{References}

[1] A.J. Niemi and G.W. Semenoff, Phys. Rev. Letts. 51 (1983) 2077;

A. N. Redlich, Phys. Rev. D29 (1984) 2366.

[2] W. Siegel, Nuc. Phys. B156 (1979) 135; J.F. Schonfeld, Nuc. Phys. B185 (1981) 157;

S. Deser, R. Jackiw and S. Templeton, Phys. Rev. Lett. 48 (1982) 975.

[3] A.J. Niemi and G.W. Semenoff, Phys. Rep. 135 (1986) 99;

I.V. Krive and A.S. Rozhavskii, Sov. Phys. Usp. 30 (1987) 370.

[4] See S.Deser, R. Jackiw and S. Templeton, Ann. Phys. (NY) 140 (1982) 372, and references therein.

[5] D. Boyanovsky, R. Blankenbecler, Phys. Rev. D31 (1985) 3234.

[6] D. Boyanovsky, R. Blankenbecler and R. Yahalom, Nuc. Phys. B270 (1986) 483.

[7] A.P. Polychronakos, Nuc. Phys. B278 (1986) 207; Phys. Rev. Letts. 60 (1988) 1920.

[8] M.B. Paranjape, Phys. Rev. D36 (1987) 3766.

[9] Y. Aharonov and D. Bohm, Phys. Rev. 115 (1959) 485;

C. R. Hagen, Phys. Rev. Letts. 64 (1990) 503.

[10] T. Jaroszewicz, Phys. Rev. D34 (1986) 3128;

Hou Bo-yu, et al, Commun. Theor. Phys. 7 (1987) 49.

[11] R. R. Parwani and A.S. Goldhaber, Nuc. Phys. B359 (1991) 483.

[12] E. G. Flekkoy and J.M. Leinaas, Int. J. Mod. Phys. A 6, 5327 (1991).

[13] V.B. Bezerra and E.R. Bezerra de Mello, Class. Quantum. Grav, 11 (1994) 457.

[14] R.D. Pisarski, Phys. Rev. D29 (1984); T. Appelquist, M. Bowick, D. Karabali, and L.C.R. Wijewardhana, Phys. Rev. D33 (1986) 3704, 3774; S. Rao and R. Yahalom, Phys. Rev. D34 (1986) 1194. M.C. Diamantini, P.Sodano and G. Semenoff, Phys. Rev. Lett. 70, 3438 (1993); K.I. Kondo, T. Ebihara, T. Iizuka and E. Tanaka, Nuc. Phys. B434 (1995) 85, and references therein.

[15] V.P. Gusynin, V.A. Miransky and I.A. Shovkovy, Phys. Rev. Letts. 73 (1994) 3499, Phys. Lett B349 (1995) 477; K.G. Klimenko, Z. Phys. C54 (1992) 323. 
[16] R. Parwani, Bhubaneswar preprint IP/BBSR/95-12, hep-th/ 9504020.

[17] Y. Aharonov and A. Casher, Phys. Rev. A19 (1979) 2461;

R. Jackiw, Phys. Rev. D 29 (1984) 2375.

[18] Ph. De Sousa Gerbert, Phys. Rev. D40 (1989) 1346.

[19] Actually because of the normalisation factor $\sim \sqrt{k}$ the scattering zero modes in (2.9 2.10) are nonvanishing as $k \rightarrow 0$ only for $|F|>1 / 2$ if $r$ is held fixed. However if $r \rightarrow 0$ much faster than $k \rightarrow 0$ then these states are nonvanishing for the full range $|F|<1$.

[20] H.N. Li, D.A. Coker and A.S. Goldhaber, Phys. Rev. D47 (1993) 694;

H.N. Li, Phys. Rev. D47 (1993) 2598.

[21] T. Banks and A. Casher, Nuc. Phys. B169 (1980) 103;

C. Vafa and E. Witten, Nuc. Phys. B234 (1984) 173.

[22] R. Mackenzie and A.M. Matheson, Phys. Lett. B259 (1991) 63.

[23] A. Niemi, Nucl. Phys. B251 (1985) 155.

[24] A. Das and M. Hott, Rochester preprint UR-1419, hep-th/9504086.

[25] A. Kovner and B. Rosenstein, Phys. Rev. B42 (1990) 4748; N. Dorey and N. E. Mavromatos, Phys. Lett. B250 (1990) 107; Nucl. Phys. B386 (1992) 614.

[26] E. M. Serbranyi, Theor. Math. Phys. 64 (1985) 846;

P. Górnicki, Ann. Phys. (N.Y.) 202, (1990) 271.

[27] A.S. Goldhaber, in "Quantum Coherence II", edited by J. Anandan and J.L. Safko (World Scientific, Singapore, 1995).

[28] A.S. Goldhaber, H.N. Li and R.R. Parwani, Phys. Rev. D51 (1995) 919.

[29] Y. Hosotani, Phys. Lett. B319 (1993) 332; D. Cangemi, E. D'Hoker and G. Dunne, Phys. Rev. D51 (1995) R2513; D. Wesolowski and Y. Hosotani, Minnesota preprint UMN-TH-1331/95, (hep-th/9505113) and references therein.

[30] I.S. Gradshteyn and I.M. Ryzhik, "Tables of Integrals, Series and Products", Academic press (NY 1980). 